\title{
Miranda
}

Revue pluridisciplinaire du monde anglophone /

Multidisciplinary peer-reviewed journal on the English-

speaking world

16 | 2018

L'expérimental dans la littérature et les arts

contemporains

\section{Thangam Ravindranathan et Antoine Traisnel, Donner le change. L'impensé animal.}

\section{Claire Cazajous-Augé}

\section{OpenEdition}

Journals

Édition électronique

URL : http://journals.openedition.org/miranda/11438

DOI : 10.4000/miranda. 11438

ISSN : 2108-6559

\section{Éditeur}

Université Toulouse - Jean Jaurès

Référence électronique

Claire Cazajous-Augé, «Thangam Ravindranathan et Antoine Traisnel, Donner le change. L'impensé animal. », Miranda [En ligne], 16 | 2018, mis en ligne le 05 juin 2018, consulté le 16 février 2021. URL : http://journals.openedition.org/miranda/11438; DOI : https://doi.org/10.4000/miranda.11438

Ce document a été généré automatiquement le 16 février 2021.

\section{(c) (i) (9)}

Miranda is licensed under a Creative Commons Attribution-NonCommercial-NoDerivatives 4.0 International License. 


\title{
Thangam Ravindranathan et Antoine Traisnel, Donner le change. L'impensé animal.
}

\author{
Claire Cazajous-Augé
}

\section{RÉFÉRENCE}

Thangam Ravindranathan et Antoine Traisnel, Donner le change. L'impensé animal (Paris : Hermann, 2016), 123 p, ISBN 978-2-7056-9213-1

1 Le titre de l'ouvrage de Thangam Ravindranathan et Antoine Traisnel, Donner le change, doit son nom à la capacité du cerf traqué à échapper à son prédateur, le chasseur, en le mettant sur la piste d'un autre membre de son espèce. Les auteurs proposent de revenir au premier sens de cette expression afin d'étudier la manière dont l'animal se dérobe à ses poursuivants, de manière littérale et métaphorique. En effet, la capacité de l'animal à donner le change inscrit un doute, un vertige, dans notre façon de chasser et dans notre façon de penser. Comment s'assurer que l'animal capturé à la fin de la partie de chasse est bien celui qui a été levé ? Afin de montrer que l'animal, être d'esquive faisant acte de duplicité, résiste à nos tentatives d'approche, de définition et de compréhension, les auteurs font appel aux textes de Derrida, d'Heidegger, de Poe, ou bien encore de Melville. Ils s'appuient sur des travaux critiques (Catherine Malabou, Philippe Jaworski, Agnès Derail-Imbert, entre autres) et proposent leurs propres analyses afin d'étudier la manière dont ces textes philosophiques et littéraires fondateurs dans le domaine de la «question animale » rendent compte de l'échappée animale.

2 La thèse centrale de cet ouvrage est que si l'animal donne le change, l'homme s'est quant à lui donné le change en s'efforçant de définir un «propre de l'homme » et en considérant l'animal comme un être qui ne ressent pas la souffrance. Ainsi, selon les auteurs, l'humain a «exilé hors de lui son impuissance et sa vulnérabilité de corps 
mortel, les rejetant sur l'animal pour, là, les méconnaître comme autant de preuves d'une privation qui ne serait pas la sienne. Chassant l'animal, au cours d'une longue histoire de la pensée humaniste, l'humain se sera donné le change» (54). Dans leur ouvrage court (environ cent pages) mais dense, Thangam Ravindranathan et Antoine Traisnel ont pour projet de rappeler aux humains leur responsabilité face à l'animal en déconstruisant l'idée d'une rupture entre le monde humain et animal.

3 Tout au long de leur ouvrage, les auteurs filent la métaphore de la chasse. Ainsi, les titres des chapitres évoquent les étapes de la traque animalière et mettent en exergue la dialectique du même et de l'autre que la chasse implique : " En défaut », «Je tue il », "Sachant chassant", ou bien encore "Fin de partie». Les auteurs recourent à un vocabulaire cynégétique technique qu'ils expliquent dans un appareillage de notes rigoureux et dans un glossaire éclairant. En outre, le texte est illustré de photographies de Jonathan Sharlin représentant différents types de "caches" de chasseurs en Nouvelle-Angleterre. Un extrait du Discours à Mme de la Sablière de Jean de la Fontaine et le poème d'Alfred de Vigny, "La Mort du loup », ouvrent et clôturent le corps du texte, et des épigraphes (extraits de textes de Benjamin, Coetzee, Kakfa, Coleridge, entre autres) introduisent chaque nouveau chapitre.

4 Après une introduction qui définit l'expression «donner le change » et qui aborde les questions qui se poseront dans les pages suivantes, le premier chapitre («En défaut») s'appuie sur la nouvelle de Poe « Double meurtre dans la rue Morgue » afin de révéler la façon dont nous omettons l'animal et le faisons disparaître de notre pensée. Dans ce texte, premier exemple de "detective fiction", personne ne s'attend à ce que le meurtrier soit un animal. L'orang-outang de la nouvelle de Poe met les enquêteurs « à défaut » («at falt »), c'est-à-dire que ces derniers perdent la trace du criminel comme les chiens de chasse sont trompés par le cerf qui donne le change. En outre, Thangam Ravindranathan et Antoine Traisnel remarquent que dans le texte original, Poe recourt à un lexique sensoriel lorsqu'il décrit les indices que Dupin, l'enquêteur, relève sur les lieux du crime. Le détective est alors à la fois un chasseur et un animal traqué, constamment aux aguets. Or, dans la traduction qu'il a faite de cette nouvelle, Baudelaire a omis la métaphore associant chasse et enquête policière qui a permis à Poe de suggérer l'idée d'une réversibilité entre homme et animal et entre chasseur et proie. Thangam Ravindranathan et Antoine Traisnel voient dans cet oubli, dans ce qu'ils nomment la « souffrance » de l'animal et de la lettre (19), un exemple frappant de notre tendance à oublier l'animal, à l'exclure de nos textes et de notre pensée.

5 Cet exemple conduit Thangam Ravindranathan et Antoine Traisnel à étudier la manière dont l'homme s'est défini en s'arrogeant un pouvoir sur l'animal, dans une logique implacable qui fait de lui un être qui «donne ordre au monde » (27). Dans le chapitre intitulé «Ergo", les auteurs reviennent sur la conjonction «donc» au centre de l'expression derridienne, «L'animal que donc je suis ». Ils insistent sur la violence de ce «donc» qui annonce de manière catégorique la séparation ou, au contraire, le lien entre l'humain et l'animal.

6 Ce qui intéresse les auteurs, c'est de savoir quel « don » peut naître de ce « donc » (27). Véritable pivot de l'ouvrage, le chapitre «Changer la donne " propose de repenser les liens entre l'humain et l'animal non pas par l'intermédiaire du « donc», mais par le "don », une autre notion étudiée par Derrida. Contrairement au «donc» qui donne lieu à des catégories fixes et fallacieuses, le don se produit sans crier gare, sans laisser de trace. Lors de la chasse, l'animal ferait don de lui ou d'un autre membre de son 
espèce. Cette notion de don ébranle l'idée que l'homme peut se définir en tant que tel en tuant l'animal, et inscrit un doute autant dans l'identité de l'animal tué que dans celle de celui qui l'a poursuivi.

7 Reprenant les propos de Derrida selon lesquels la philosophie échoue à penser l'animal, Thangam Ravindranathan et Antoine Traisnel se penchent ensuite sur la capacité de la poésie à dire l'indicible et l'inconnaissable qu'est l'animal. Les auteurs sollicitent des textes de Ponge, de Michaux ou de Melville afin de suggérer que la poésie serait la " charnière » entre la chair et les mots car elle permet de relier, contre toute logique, des éléments en apparence opposés : fiction et réalité, vie et mort, humain et animal, prédateur et proie (48). Comme le leurre que l'homme tient dans sa main ou au bout de sa ligne et vers lequel l'animal se dirige, la poésie est un lieu de rencontre entre l'humain et l'animal. Avec le leurre, et donc avec la poésie, les identités se confondent parfois, glissant l'une vers l'autre: "qui leurre ? qui est leurré ? ce qui revient à demander : qui donne ? qui prend ?» (51). Ainsi, plutôt que de nommer l'animal, ce qui pourrait s'apparenter à un acte de prédation verbale, certains auteurs choisissent de recourir à des stratégies narratives et descriptives qui restituent la confusion entre le chasseur et l'animal, comme lorsque Cendrars, dans son poème "Chasse à l'éléphant », utilise le pronom «il » qui pourrait aussi bien désigner l'humain que le pachyderme.

guise de conclusion, Thangam Ravindranathan et Antoine Traisnel rappellent leur thèse principale : en se présentant comme un être supérieur à l'animal, l'homme s'est donné le change. La présence d'un troisième membre dans la partie de chasse, le chien, contribue à montrer que la relation chasseur-animal ne saurait être envisagée comme un simple duel. Le chien joue un rôle d'auxiliaire qui partage ses sens et ses savoirs avec l'homme. Il est à la fois chasseur et chassé, un "chassant» (81) qui est capable de capturer la proie mais aussi susceptible d'être leurré par le cerf. Thangam Ravindranathan et Antoine Traisnel jouent alors sur le sens du mot « auxiliaire » : « Si c'est lui qui donne (ou fait perdre) la proie au chasseur, c'est lui aussi qui donne (ou fait perdre) la chose au langage»(82). Dans un retournement final, les auteurs se demandent si l'auxiliaire ne serait pas la véritable question posée par Derrida, nous invitant une dernière fois à relire L'Animal que donc je suis à la lumière de ces nouvelles pistes.

9 Dans le foisonnement de publications consacrées à la question animale, cet ouvrage éclairant et stimulant constitue une contribution essentielle à la réflexion sur « l'impensé » et « l'impensable » animal. Thangam Ravindranathan et Antoine Traisnel font dialoguer avec élégance et érudition des théories de penseurs de l'animal, des textes littéraires et des extraits d'ouvrages de vénerie et, dans un véritable esprit derridien, interrogent les plis du langage poétique et ordinaire afin d'y déceler le don que nous fait l'animal. 
INDEX

Keywords : animal, hunting, philosophy

Mots-clés : animal, chasse, philosophie

\section{AUTEURS}

\section{CLAIRE CAZAJOUS-AUGÉ}

Prag

Université Toulouse 2 - Jean Jaurès

claire.cazajous@univ-tlse2.fr 